\title{
CONSEQUENCES OF EATING LIPIDS
}

William E.M. Lands, Division of Basic Research, National Institute on Alcohol Abuse and Alcoholism, Rockville, MD 20857, USA

Thrombosis and arrhythmia cause morbidity and mortality for many Americans, for whom nearly half of all deaths are due to cardio- (or cerebro-) vascular complications. The marked differences in age-specific death rates between the USA and Japan make it important for Americans to learn from the Japanese biomedical scientists how Japanese maintain a lower risk of chronic and acute factors contributing to cardiovascular death. Researchers have considered differences in life-style, including smoking and alcohol intake, as well as the type and amount of dietary lipid. Members of the Japan Society for Lipid Nutrition are well qualified to evaluate the way that acute thromboembolic complications are mediated by a rapid over-production of the n- 6 eicosanoid, thromboxane, and cardiac arrhythmias are mediated by $n-6$ eicosanoid-enhanced adrenergic signaling. Either thrombosis or arrhythmia can convert a chronic stenotic condition into an acute ischemic condition, and the role of lipid nutrition in the origins of both conditions needs interpretation.

For chronic stenosis, the importance of suppressing the HMG-CoA reductase-catalyzed conversion of dietary materials into mevalonate, isoprenoids and cholesterol is documented extensively with quantitative correlations of plasma cholesterol with dietary statuated fats and cardiovascular mortality, but cellular mediators linking cholesterol to stenosis and mortality remain poorly defined. In contrast, for acute ischemia, the importance of cellular mediators (prostaglandins, thromboxane and leukotrienes) formed from dietary n- 6 fatty acids is documented in mediating thrombosis and arrhythmia, but quantitative correlations linking tissue levels of eicosanoid precursors to dietary fats need to be defined. Our results suggest that the capacity for a tissue response mediated by $n-6$ eicosanoids can be related to the percent of highly unsaturated fatty acids of phospholipids that are in the form of the $n-6$ eicosanoid precursors, 20:3+20:4n-6. 
Empirical hyperbolic equations were developed from dietary studies with rats [Lipids 25:505 (1990)] to describe the competitions between the n-3 and n6 types of highly unsaturated fatty acids which influence the proportion of $n-6$ eicosanoid precursors maintained in the phospholipids of a tissue. The equations were developed by categorizing fatty acids in accord with general acyltransferase specificities. The categories include saturated fatty acid (SFA; $14: 0,16: 0,18: 0), 16-$ and 18-carbon unsaturated fatty acids (UFA;16:1n-7, $18: 1 \mathrm{n}-7,18: 1 \mathrm{n}-9,18: 2 \mathrm{n}-6,18: 3 \mathrm{n}-3)$, and 20-and 22-carbon highly unsaturated fatty acids (HUFA; 20:3n-9, 20:4n-7, 20:3n-6, 20:4n-6, 22:4n-6, 22:5n-6, 20:5n-3, 22:5n-3, 22:6n-3). De novo synthesis of glycerolipids places SFA at position 1 and UFA at position 2, providing phospholipids with nearly $50 \%$ of the fatty acids as SFA and triglycerides with about 33\% SFA. Retailoring acyltransferases replace some UFA at position 2 with HUFA, maintaining an average general fatty acid composition of phospholipids of about $43 \%$ SAF, $36 \%$ UFA and $20 \%$ HUFA in the plasma of rats and humans. Because retailoring acyltransferases are relatively non-selective with regard to $n-3, n-6$, $n-7$, and $n-9$ chemistry of the fatty acids, the dietary supply of different types of HUFA strongly affects the proportion of HUFA that is maintained in a tissue in the form of the $n-6$ eicosanoid precursors, 20:3 + 20:4n-6 [Annu. Rev. Nutr. 11, 41(1991)].

Dietary intakes of different fatty acids can be used with the new equations to predict the proportions of eicosanoid precursors that will be maintained in plasma phosholipids of humans. Alternatively, the equations may be used in reverse to estimate the dietary intakes of the $n-3$ and $n-6$ fatty acids from the composition of the fatty acids that has been maintained in plasma lipids. The empirical equations successfully relate dietary data to clinical and epidemiological data. For example, a large prospective study showed that intake of n-3 fatty acids was related to less relative risk independent of age, race, smoking, diastolic blood pressure, LDL or $\mathrm{HDL}$ cholesterol, and that Americans ingesting about $0.66 \mathrm{~g} n-3$ HUFA per day had only $60 \%$ of the relative risk for cardiovascular death than average American men (who ingested daily only ca. $0.05 \mathrm{~g} \mathrm{n}-3$ HUFA). Ischemic heart disease mortality for Japanese 
ingesting approximately $3 g$ n-3 HUFA per day was only $20 \%$ of the typical USA rate, and it was still lower for Greenlanders whose diets contained nearly $10 \mathrm{~g} n-3$ HUFA per day. The new equations help link quantitatively the dietary intakes of $n-3$ and $n-6$ fatty acids to the levels of $n-6$ eicosanoid precursors maintained in tissues, to the known frequency and severity of important eicosanoid-mediated diseases, and also to existing epidemiological data on morbidity and mortality [FASEB J. 6:2530 (1992)]. More careful longitudinal studies in Japan seem certain to provide the world with more important information on the relation of lipid nutrition to health and disease. 